\title{
Subpicosecond photoinduced Stark spectroscopy in fullerene-based devices
}

\author{
J. Cabanillas-Gonzalez, T. Virgili, A. Gambetta, L. Lüer, and G. Lanzani \\ IFN-CNR, Dipartimento di Fisica, ULTRAS-INFM, Politecnico di Milano, Milano 20133, Italy
}

T. D. Anthopoulos

Blackett Laboratory, Department of Physics, Imperial College London, London SW7 2BW, United Kingdom

D. M. de Leeuw

Philips Research Laboratories, Professor Holstlaan 4 (WAG 11), 5656 AA Eindhoven, The Netherlands

(Received 24 July 2006; published 18 January 2007)

\begin{abstract}
We present a detailed study of the charge photogeneration and decay kinetics in [6,6]-phenyl C61-butyric acid methyl ester by performing subpicosecond Stark spectroscopy measurements. Owing to the large photogenerated charge density and the strength of the applied field, progressive quenching of the Stark signal is observed during the first $20 \mathrm{ps}$, followed by a slow recovery at longer time scales. The quenching regime provides information on the average $e-h$ separation and drift motion within the $e-h$ pair whereas the recovery is related to the kinetics of charges and their recombination probability.

DOI: 10.1103/PhysRevB.75.045207

PACS number(s): 78.47.+p, 73.61.Wp, 73.23.-b, 78.20.-e
\end{abstract}

\section{INTRODUCTION}

The power conversion efficiency of organic photovoltaic cells is mainly determined by the charge photogeneration (CPG) quantum yield of the semiconductor and the charge collection efficiency at the electrode terminals of the device. The former is given by the fraction of absorbed photons converted into charge carriers whereas the latter takes into account the probability of these carriers to be transported and collected by the corresponding electrodes. CPG quantum yields close to unity have been demonstrated in a number of bulk heterojunction organic solar cells based on interpenetrating networks of a hole transporting polymer, such as $\mathrm{OC}_{1} \mathrm{C}_{10} \mathrm{PPV}$, and an electron accepting organic molecule, such as solution processible $C_{60}$ fullerene derivatives. ${ }^{1,2}$ Based on these findings it is now widely accepted that one of the main limitations in the power conversion efficiency of organic photovoltaic devices is the moderate transport of the photogenerated charges, i.e., holes and electrons, toward the corresponding collecting electrodes. There are two main mechanisms responsible for the relatively poor charge carrier transport: carrier trapping and carrier recombination. Trapping can occur due to chemical impurities present within the material and/or to conformational defects in the percolation path. Recombination of opposite sign charges is rather efficient due to the fairly weak screening in organics $(\varepsilon \sim 4)$ as compared to that in inorganic semiconductors $(\varepsilon \sim 10)$, which enhances Coulomb capture radius (about $20 \mathrm{~nm}$ ) and reduces the probability of escaping from geminate recombination. In this context it has been reported that the highest efficiencies are found in devices containing large weight percentage of the $C_{60}$ soluble derivative [6,6]-phenyl C61butyric acid methylester (PCBM) distributed as aggregates within the polymeric bulk. ${ }^{3,4}$ The beneficial properties appear to be related with large mobility values exhibited inside these aggregates. ${ }^{5,6}$ Moreover, field affect transistors based on various PCBM derivatives $\left(\mathrm{C}_{60^{-}}, \mathrm{C}_{70^{-}}\right.$, and $\left.\mathrm{C}_{84^{-}} \mathrm{PCBM}\right)$ exhibit ambipolar charge transport. ${ }^{7-9}$ Making use of this prop- erty the first complementarylike logic circuits have been recently demonstrated. ${ }^{8,10}$ Clearly molecular properties, intermolecular interactions, morphology, and composition strongly affect transport. Experimental techniques for measuring mobility and carrier kinetics in different regimes and time domains are thus demanded in order to assess models and enhance tailoring and design capabilities of new materials with improved transport properties.

In this work we monitor field induced singlet dissociation and charge recombination in $C_{60}$-PCBM sandwich cells with subpicosecond time resolved photoinduced Stark spectroscopy. This technique traces for changes on the electroabsorption $(E A)$ signal induced by photogenerated charges. Upon impulsive photogeneration in the presence of an internal field, singlets dissociate into charge pairs (Fig. 1). These pairs orient along the field axis and drift in opposite directions, increasing their associated dipole moment and leading to a net photoinduced polarization which progressively

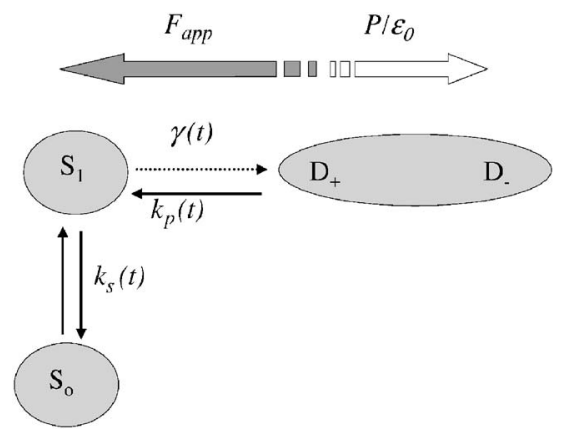

FIG. 1. The CPG process under an $F_{a p p}$ applied electric field. Photogeneration leads to formation of $S_{1}$ singlet excited states which can either dissociate into charge pairs with a $\gamma$ dissociation rate or decay with a rate given by $k_{s}$. As a result of the applied field, pairs get oriented along the field axis and the $e-h$ separation increases progressively leading to a photoinduced polarization $P$ which opposes to the applied field. Eventually, charge pairs recombine with a rate $k_{p}$ to yield new $S_{1}$ states. 
screens the internal field. Evolution of the internal field upon photogeneration is a clear proof that the field is being modified by photogenerated charges since effects due to injected charges would otherwise be independent on the photogeneration process. Recovery of the internal field takes place only when a fraction of charges recombine. Therefore by monitoring the internal field through the EA signal during the process of CPG, it is possible to obtain a unique picture of both charge motion and kinetics. Here we are mainly concerned with the follow-up dynamics until about $0.5 \mathrm{~ns}$, focusing our attention on the evolution of neutral and charge states and their recombination processes. Results and discussion are an extension of a previous short paper, which was focused on mobility determination from the early time dynamics. ${ }^{11}$ Our results suggest that geminate recombination is responsible for a loss of $40 \%$ of the initially photogenerated charges in the first $450 \mathrm{ps}$.

\section{EXPERIMENTAL DETAILS}

\section{A. Sample preparation and description of method}

Encapsulated single layer PCBM diodes were fabricated in glove box conditions by spincoating $60 \mathrm{~g} / 1$ chlorobenzene solutions of PCBM with $0.7 \%$ weight content of polystyrene over polyethylenedioxythiophene coated indium tin oxide (ITO) substrates at $1600 \mathrm{rpm}$. Typical thickness of the spin coated films was between 113 and $120 \mathrm{~nm}$. Gold electrodes were then thermally evaporated on top of the films through a shadow mask to produce four pixels of different size ranging from $3 \times 3 \mathrm{~mm}$ to $10 \times 10 \mathrm{~mm}$. Current density-voltage $(J-V)$ characterization was carried out with a Keithley 236 source measure unit under ambient conditions. Steady-state electromodulation spectroscopy was performed with a $30 \mathrm{~W}$ Spectral Products tungsten lamp coupled to a monochromator where the light output was directed to one of the pixel devices through the substrate (glass/ITO) side. The light reflected back from the cathode was focused using a lens onto a silicon based photodiode connected to a Stanford Instruments lock-in amplifier referred to the frequency of the applied electric field. An alternating electric field of $1 \mathrm{MV} / \mathrm{cm}$ amplitude at $470 \mathrm{~Hz}$ was applied across the device being the experiment was carried out at room temperature. The pumpprobe setup is based on an amplified Ti:sapphire laser delivering $780 \mathrm{~nm}$ pulses of $150 \mathrm{fs}$ at $1 \mathrm{KHz}$ repetition rate and $750 \mu \mathrm{J}$ output power. The fundamental beam was split into pump and probe, the pump being doubled in frequency to produce $390 \mathrm{~nm}$ excitation pulses with fluence comprised between 0.08 and $0.3 \mathrm{~mJ} \mathrm{~cm}^{-2}$ Pump pulses were sent to a delay line and focused on one pixel through the transparent ITO side. The probe beam was focused onto a sapphire plate to generate a supercontinuum pulse and spatially overlapped with the pump on the pixel. Detection was provided by a silicon based photodiode with an interferometer filter for wavelength selection of the probe reflected back the gold cathode surface. Optical pump-probe measurements were performed in the absence of electric field by mechanically chopping the pump at $470 \mathrm{~Hz}$ and detecting the probe with the aim of a lock-in amplifier referred to the chopping frequency. For electro-optic pump-probe measurements, a square profile electric field modulated at $470 \mathrm{~Hz}$ between 0 and $1.5 \mathrm{MV} / \mathrm{cm}$ was applied in reverse configuration to minimize charge carrier injection. Phase-sensitive detection was achieved with the aim of a lock-in amplifier referred to the frequency of the field.

\section{B. Physical meaning of the pump-probe signal}

The optical pump-probe signal or differential transmission $(\Delta T / T)$ depends on energy $(\omega)$ and delay time $(\tau)$ and is given by:

$$
\Delta T / T(\omega, \tau)=-d \sum_{i j} \sigma_{i j}(\omega) \Delta N_{j}(\tau) \otimes f_{p}
$$

being $d$ the thickness of the film, $\sigma_{i j}$ the cross section for the $j \rightarrow i$ excited state transition, $\Delta N_{j}$ the pump induced change in the population of $j$ states, and $f_{p}$ the normalized probe pulse temporal profile. $\Delta T / T$ thereby provides the temporal evolution of the $N_{j}$ photoexcited states. The electro-optic pump-probe signal collected under electric field modulation $\left(\Delta^{2} T / T\right)$, measures the field induced changes in $\Delta T / T$, which taking into account Eq. (1) is given by (Ref. 12):

$$
\begin{aligned}
\Delta^{2} T / T= & \Delta T /\left.T\right|_{F}-\Delta T / T \\
= & -d \sum_{i, j} \Delta \sigma_{i j}(\omega, F) \Delta N_{J} \otimes f_{p}-d \sum_{i, j} \sigma_{i j}(\omega) \Delta^{2} N_{J}(F) \\
& \otimes f_{p}-\sum_{i} \Delta \sigma_{i o}(F) N_{o},
\end{aligned}
$$

where the three terms from left to right correspond to the Stark shift of the excited state transition, field induced changes in the population of $j$ states, and ground state Stark shift, respectively. In the absence of $E A$ signal, $\Delta^{2} T / T$ provides information exclusively on the evolution of $j$ states under the electric field. In this case, the sign of $\Delta^{2} T / T$ indicates field induced increase or reduction of $j$ states, depending on the sign of $\Delta T / T$ (e.g., whether $\Delta T / T<0, \Delta^{2} T / T$ $<0$ holds for increase in population whereas $\Delta^{2} T / T>0$ indicates population quenching). Concerning the ground state $E A$ contribution, this term depends only on the internal electric field $(F)$. On the basis of constant $F$, it will only provide a time independent flat offset, since the population of the ground state $\left(N_{0}\right)$ is essentially constant in time. However, we will show in this work that the internal field is modified during the CPG process, leading therefore to additional temporal dependency in the ground state $E A$ term.

\section{RESULTS}

\section{A. Steady-state electromodulation spectra}

Figure 2 displays the steady state $(\mathrm{CW})$ electromodulated spectra of PCBM at room temperature. For an applied bias of the type $F_{d c}+F_{a c} \sin \omega$ t, the $E A$ signal can be approximated as follows: 


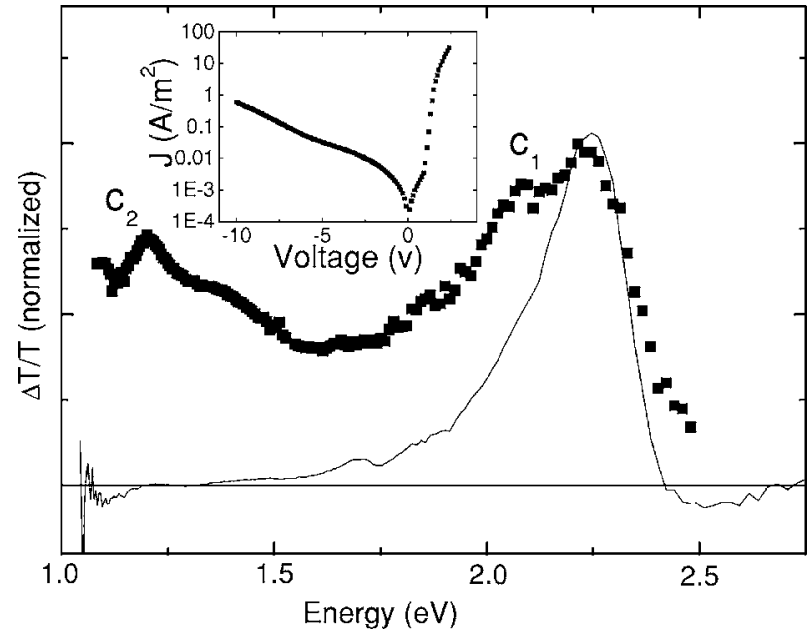

FIG. 2. Electromodulated spectra at room temperature for an applied field of $F_{\mathrm{dc}}=0$ and $F_{\mathrm{ac}}=1 \mathrm{MV} / \mathrm{cm}$ and detecting at $2 \omega$ (solid line), and $F_{\mathrm{dc}}=0.5 \mathrm{MV} / \mathrm{cm} F_{\mathrm{ac}}=0.5 \mathrm{MV} / \mathrm{cm}$ and detecting $1 \omega$ (solid squares). The inset displays the $J$ - $V$ characteristics of a PCBM diode.

$$
\begin{aligned}
E A_{i j}(\omega) \approx & \Delta p_{i j}(\omega) F_{i}^{2} \\
\approx & \Delta p_{i j}(\omega)\left[\frac{1}{2} F_{a c}^{2}+F_{d c}^{2}+2 F_{a c} F_{d c} \sin \omega t\right. \\
& \left.+\frac{1}{2} F_{a c}^{2} \sin 2 \omega t\right],
\end{aligned}
$$

where $\Delta p_{i j}(\omega)$ is the change in the polarization tensor component $i j$ induced by the field. We have applied the electric field in two different configurations:

(a) $F_{d c}=0$ and $F_{a c}=1 \mathrm{MV} / \mathrm{cm}$; (b) $F_{d c}=0.5 \mathrm{MV} / \mathrm{cm}$ and $F_{a c}=0.5 \mathrm{MV} / \mathrm{cm}$.

In the first case the only time dependent term scales with $\sin 2 \omega \mathrm{t}$ so that the spectrum recorded at $2 \omega$ has exclusive contribution from $\Delta p(\omega)$. As it can be observed in Fig. 2, the $2 \omega$ spectrum is characterized by a sharp feature with maximum at $2.3 \mathrm{eV}$. Assignment of this feature in crystalline $C_{60}$ is not clear although some reports attribute it to a charge transfer exciton delocalized over two nearest neighbor $C_{60}$ molecules. In PCBM we reported previously a quadratic dependence with the applied electric field characteristic of excitonic Stark shift. ${ }^{11}$ In the second case, the signal depends on $1 \omega$ as well as $2 \omega$. In addition to $E A$, signatures from charge induced absorption (bands $c_{1}$ and $c_{2}$ ), are present at $1 \omega$, as expected from the high rectification ratio of the $J-V$ diode curve and the large charge injection achieved at positive bias (inset Fig. 2). In PCBM charge absorption is characterized by two main bands: one with peak at $1.2 \mathrm{eV}$ and shoulder at $1.35 \mathrm{eV}$, and a weaker band located at $2 \mathrm{eV}$. Our data is perfectly consistent with previa assignments of the absorption of fullerene anions ${ }^{13}$ and photoinduced absorption $(P A)$ spectroscopy at room temperature in $C_{60}$ films reported by Vardeny and co-workers. ${ }^{14}$ In this latter work, these two bands are assigned to polaron subgap and intragap transitions, respectively.

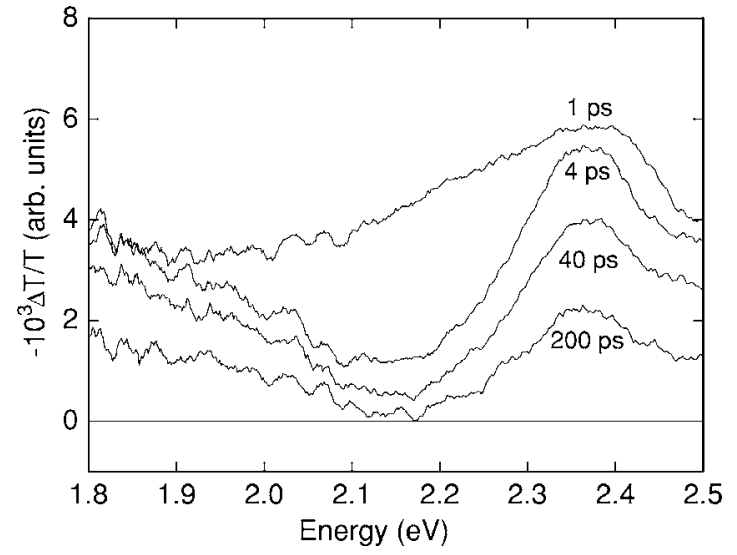

FIG. 3. Pump-probe spectra without electric field at delay times from 1 to 200 ps.

\section{B. Pump-probe spectra and dynamics}

The pump-probe spectra shown in Fig. 3 are characterized by two PA bands: one centered at $2.38 \mathrm{eV}$ and a broader band which extends beyond $1.8 \mathrm{eV}$. The spectral region between $1.9-2.4 \mathrm{eV}$ is characterized by a fast decay component during the first $4 \mathrm{ps}$, leading to a narrowing of the $P A$ bandwidth, which loses oscillator strength on the lower energy tail. A deeper insight is provided by the $P A$ dynamics at 1.2, 1.8, and $2.3 \mathrm{eV}$ [Fig. 4(a)]. Here also the different behavior at $2.3 \mathrm{eV}$ is evident. Taking into account that photoexcitation takes place at $3.2 \mathrm{eV}$ and that PCBM optical gap is located at $2.7 \mathrm{eV}\left(S_{2}\right)$ [neglecting the first forbidden state at $1.8 \mathrm{eV}$ (Ref. 15)], the observed fast $P A$ dynamics can be assigned to energy relaxation to the lowest excited state. Both internal conversion $S_{2}-S_{1}$ and vibrational relaxation are taking place. The former process is expected to proceed in the 100 fs time domain for large organic molecules. ${ }^{16-18}$ In addition, $S_{2}-S_{n}$ transitions would lead to dramatic change of the $P A$ spectrum with respect to $S_{1}-S_{m}$ (note that $S_{n}$ and $S_{m}$ have different symmetry). Therefore it seems more reasonable to assign the ps dynamics to hot $S_{1}^{*}$ state thermalization.

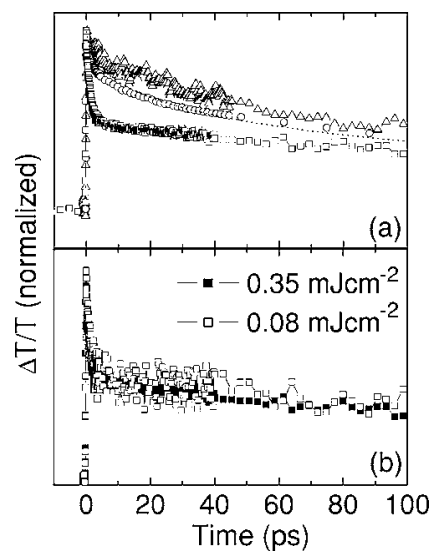

FIG. 4. (a) PA dynamics at $2.3 \mathrm{eV}$ (squares), $1.8 \mathrm{eV}$ (circles), and $1.2 \mathrm{eV}$ (triangles) pumping with $0.35 \mathrm{~mJ} \mathrm{~cm}^{-2}$. The $1.8 \mathrm{eV}$ dynamics was fitted to a stretch exponential law (dotted line). (b) PA dynamics at $2.3 \mathrm{eV}$ under two different pump fluences of $0.35 \mathrm{~mJ} \mathrm{~cm}^{-2}$ (filled squares) and $0.08 \mathrm{~mJ} \mathrm{~cm}^{-2}$ (open squares). 


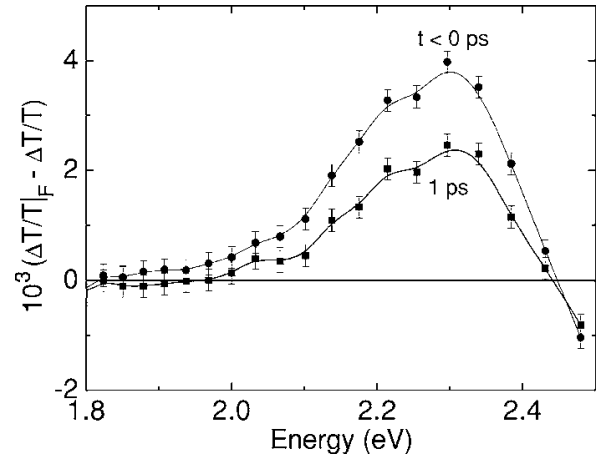

FIG. 5. Electric field pump-probe spectra at negative (circles) and 1 ps (squares) delays.

Following $S_{2}-S_{1}$ internal conversion, some energy is apparently stored into optically active vibrational modes and give rise to the redshift absorption tail. After few ps the dynamics at $2.3 \mathrm{eV}$ slows down and follow parallel trends to 1.8 and $1.2 \mathrm{eV}$. Hereby the most likely explanation is that the relaxation from $S_{1}^{*}$ to $S_{1}$ is completed and all dynamics reflect the temporal evolution of $S_{1}$. It is also noteworthy that we have not found traces of bimolecular recombination: the dynamics at $2.3 \mathrm{eV}$ pumping with intensities in the range from $0.08 \mathrm{~mJ} \mathrm{~cm}^{-2}$ to $0.35 \mathrm{~mJ} \mathrm{~cm}^{-2}$ show the same kinetics [Fig. 4(b)]. Similar studies reported by Dexheimer et al. in $C_{60}$ crystalline films have demonstrated a certain intensitydependent behavior on the dynamics of the lowest $S_{1}$ state, although in that case the excitation regime employed was one order of magnitude higher with respect to our studies. ${ }^{19}$

\section{Electro-optic pump-probe spectra and dynamics}

The electric field pump-probe spectra are provided in Fig. 5. The spectrum at negative pump-probe delay (pump arrives after probe) is independent of the pump. It reflects electromodulation of the probe and therefore its shape resembles $E A(\omega)$. Since the electric field is applied in reverse bias, charge injection is minimized according to the large rectification ratio of the diodes leading to an absence of charge induced features in the low energy part of the spectrum compared with Fig. 2. At 1 ps pump-probe delay, $\Delta T /\left.T\right|_{F}$ $-\Delta T / T$ is already reduced to nearly $60 \%$ its initial value. There is no evidence in the spectra of new additional features suggesting that the signal is still dominated by $E A$. The electric field pump-probe dynamics at three different energies: $2.3,2.0$, and $1.8 \mathrm{eV}$ are provided in the 40 and $400 \mathrm{ps}$ time range in Figs. 6(a) and 6(b), respectively. Concerning the short time scale, the $2.3 \mathrm{eV}$ dynamics exhibits a flat background at negative delays due to $E A$. At positive delays, $E A$ is quenched progressively toward $20 \%$ its original value during the first $10 \mathrm{ps}$. At $2.0 \mathrm{eV}$ there is still $E A$ contribution although superposed with a weak steplike signal which does not influence significantly the overall dynamics. On the contrary the $1.8 \mathrm{eV}$ dynamics shows negligible $E A$ (see spectrum in Fig. 5), and the only contribution is given by the steplike positive signal. This must therefore be associated to field induced population changes. Since at zero field $P A$ is assigned to $S_{1}$ absorption, the positive $\Delta T /\left.T\right|_{F}-\Delta T / T$ points to population reduction due to singlet dissociation under the applied field, which appears to occur instantaneously (i.e., within the pump pulse). The long scale dynamics, however, show a slight recovery of the $E A$ signal both at 2.3 and $2.0 \mathrm{eV}$ [Fig. 6(b)], and correspondingly the $1.8 \mathrm{eV}$ reflects a slow recovery in the singlet population. Such phenomenon could be the result of charge recombination, which might extend beyond the 400 ps time range, an issue which will be addressed in the next paragraph. Finally we focus our attention on the dynamics at $2.3 \mathrm{eV}$ exciting at high and low pump fluence provided in Fig. 7. Here it can be observed how the recovery of the EA signal is independent of the excitation density. This observation seems to exclude bimolecular, nongeminate charge recombination as the cause for EA recovery.

\section{ANALYSIS AND DISCUSSION}

In the following we proceed to describe the model employed for fitting the experimental data, being the simulated traces displayed in Figs. 4(a), 6(a), and 6(b).

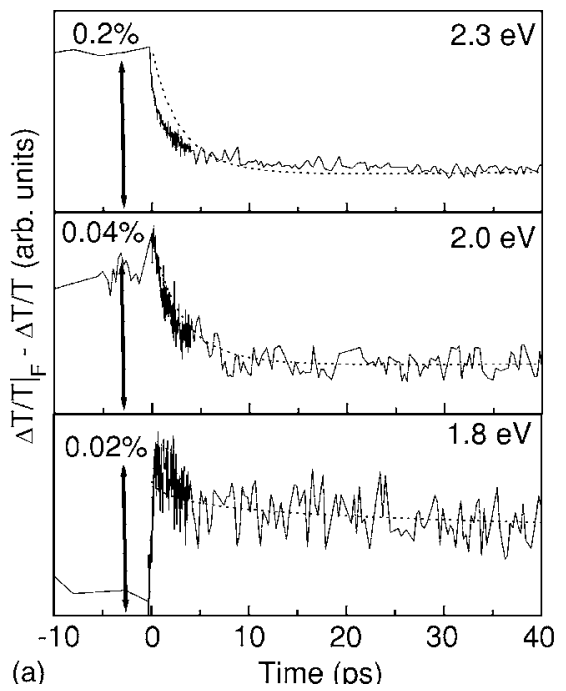

(a)

Time (Ds)

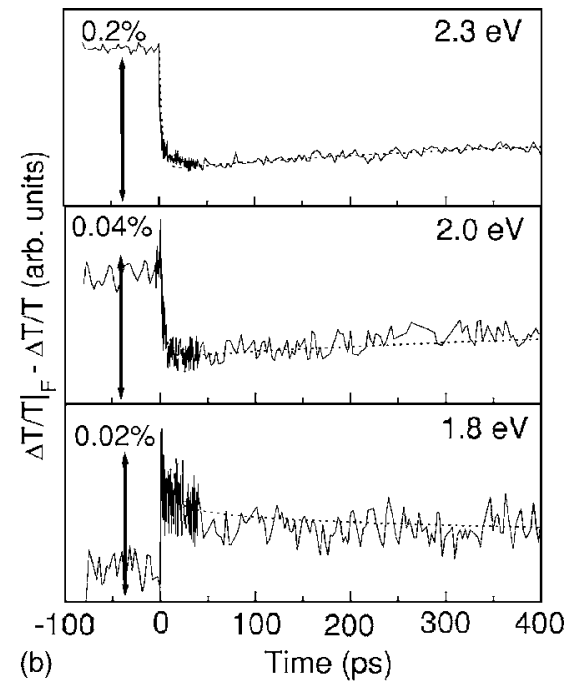

045207-4
FIG. 6. Electric field pumpprobe dynamics at $2.3,2.0$, and $1.8 \mathrm{eV}$ (a) in the $40 \mathrm{ps}$ and (b) 400 ps time window. For comparison purposes, the $Y$ axis is enlarged five times for the $2.0 \mathrm{eV}$ dynamics and 10 times for the $1.8 \mathrm{eV}$ dynamics being the amplitude signals provided in percentage values. Fits obtained from data analysis are shown for each dynamic (dotted line). 


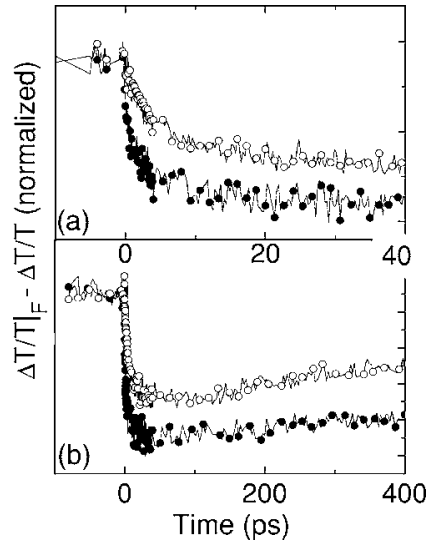

FIG. 7. Electric field pump-probe dynamics at $2.3 \mathrm{eV}$ (a) in the $40 \mathrm{ps}$ and (b) $400 \mathrm{ps}$ time window exciting with $0.35 \mathrm{~mJ} \mathrm{~cm}^{-2}$ (filled circles) and $0.08 \mathrm{~mJ} \mathrm{~cm}^{-2}$ (open circles). Note that both follow parallel trends.

\section{A. Singlet population}

In the absence of electric field and neglecting the effects of singlet-singlet annihilation, the singlet population density evolves as $N_{s}=N_{s}(0) e^{-k_{s} t}$ where $N_{s}(0)$ is the initial singlet density determined from the pump fluence and the absorbance of the film and $k_{S}$ the monomolecular recombination rate. Assuming exclusive singlet contribution, $\Delta T / T$ at $1.8 \mathrm{eV}$ can therefore be fitted as follows:

$$
\frac{\Delta T^{1.8 \mathrm{eV}}}{T}=\sigma^{1.8 \mathrm{eV}} N_{s}(0) e^{-k_{s} t} d
$$

being $\sigma^{1.8 \mathrm{eV}}$ and $d$ the cross section at $1.8 \mathrm{eV}$ for the $S_{1^{-}}$ $S_{m}$ transition and the film thickness, respectively. The population of singlets under an applied electric field $\left(\left.N_{S}\right|_{F}\right)$ is governed by:

$$
\frac{\left.d N_{s}\right|_{F}}{d t}=-\left.k_{s} N_{s}\right|_{F}-\left.\gamma N_{s}\right|_{F}+k_{p} N_{p},
$$

where two additional contributions appear, a negative term $\left(-\left.\gamma N_{s}\right|_{F}\right)$ due to dissociation of singlets into charge pairs with a dissociation rate $\gamma$, and a positive term $\left(k_{p} N_{p}\right)$ due to singlet regeneration from charge recombination with a recombination rate $k_{p} . \gamma$ is found to be important within the pulse duration and decrease dramatically after, as it appears from the saturation of field induced singlet dissociation beyond 1 ps displayed in Fig. 6(a). Such behavior is likely motivated by the high probability of dissociation of hot $S_{1}^{*}$ states and in agreement with the thermalization time scale of few picoseconds that we inferred. We remark, however, that dissociation of thermalized $S_{1}$ states cannot be ruled out since the dynamics of singlets with and without field diverge on the long time scale. Hereby, both $\gamma$ and $k_{p}$ are unknown and cannot be univocally determined simply by fitting the electric field dynamics at $1.8 \mathrm{eV}$ to Eq. (5). We therefore need to disentangle $k_{p}$ from $\left.N_{s}\right|_{F}$. Hitherto there are no traces of polaron absorption in the field induced dynamics (the signal is dominated by $E A$ as well as singlet contribution), $k_{p}$ can be directly determined from the recovery of the $E A$ signal at long delays.

\section{B. Charge population}

The expression for the population of charge pairs generated by the field is given by:

$$
\frac{d N_{p}}{d t}=-k_{p} N_{p}+\left.\gamma N_{s}\right|_{F}
$$

According to the expression described for the singlet population, an $e-h$ pair forms from singlet breaking, leading to a charge pair creation rate of $\left.\gamma N_{s}\right|_{F}$. The charge recombination rate $-k_{p} N_{p}$ describes a monomolecular recombination of charge pairs to form a new singlet state. We remark here that we have considered geminate recombination to be the most likely scenario. This assumption is based on three different observations: first, the lack of intensity dependence observed in the $E A$ dynamics depicted in Fig. 7. Second the average $e-h$ separation distance estimated under the same conditions, which is not superior to $5 \mathrm{~nm}$, according to our previous studies $^{11}$ and therefore far below the $15 \mathrm{~nm}$ estimated for the Coulomb capture radius at room temperature. According to this scenario, the probability for a charge to escape from the mutual attractive Coulombic interaction is very low. Third, the average separation between geminate pairs is $6.2 \mathrm{~nm}$ according to the initial charge pair density $\left(2.3 \times 10^{18} \mathrm{~cm}^{-3}\right)$ and based on the hard sphere approximation. This value is larger than the average $e$ - $h$ distance estimated from polarization, so we can rule out interaction between pairs.

\section{Photoinduced transient Stark signal}

In this section we address the reduction of the Stark signal induced by the pump. The magnitude of $E A$ quenching is nearly $80 \%$ and by monitoring its evolution in real time it is possible to infer the average $e$ - $h$ separation $\langle R(t)\rangle$ along the field axis as well as the charge kinetics. On account of Eq. (3) $E A$ can be approximated as $E A(t) \approx \alpha F_{\text {eff }}^{2}$ where $F_{\text {eff }}$ is the effective field inside the photogenerated area and $\alpha$ is proportional to the change in polarizability induced by the field and estimated to be $1.3 \times 10^{-3} \mathrm{~cm}^{2} / \mathrm{MV}^{2}$ in our previous publication. ${ }^{11} F_{\text {eff }}$ is given by:

$$
F_{\text {eff }}=F_{\text {app }}-\frac{\langle P(t)\rangle}{\varepsilon_{o}}=F_{a p p}-\frac{e N_{p}(t)\langle R(t)\rangle}{\varepsilon_{o}},
$$

where $\langle P(t)\rangle$ is the projection of the photoinduced polarization along the field axis, which is proportional to the product of $N_{p}(t)$ and the average $e-h$ distance in the field axis $\langle R(t)\rangle$. This latter parameter is responsible for the progressive decrease of the $E A$ signal which beyond 20 ps is overcome by slow charge recombination and therefore weak $E A$ recovery. Here we note that if we include in $N_{p}(t)$ charge pairs generated gradually as well as those created during pump excitation, determination of $F_{\text {eff }}$ becomes complicated since it would require one to consider drift events starting at different time intervals. In this study we will consider that only drift of $e$ - $h$ pairs photogenerated within the pump pulse con- 
TABLE I. Parameter values employe to fit the experimental data.

\begin{tabular}{|c|c|c|c|}
\hline$N_{s}(0)\left(\mathrm{cm}^{-3}\right)$ & $N_{p}(0)\left(\mathrm{cm}^{-3}\right)$ & $k_{s}\left(\mathrm{ps}^{-1}\right)$ & $k_{p}\left(\mathrm{ps}^{-1}\right)$ \\
\hline $3.1 \times 10^{19}$ & $2.3 \times 10^{18}$ & $6.2 \times 10^{-2} t^{-0.59}$ & $6 \times 10^{-3} t^{-0.5}$ \\
\hline$\sigma^{1.8 \mathrm{eV}}\left(\mathrm{cm}^{2}\right)$ & $\sigma^{2.0 \mathrm{eV}}$ & $\gamma\left(\mathrm{ps}^{-1}\right)$ & $\langle r(t)\rangle_{\mathrm{drift}}(\mathrm{nm})$ \\
\hline $1.0 \times 10^{-17}$ & $0.18 \times 10^{-17}$ & $8 \times 10^{-4} t^{-0.1}$ & $3[1-\exp (-t / 5)]$ \\
\hline
\end{tabular}

tribute to screen the applied electric field, i.e., $N_{p}(t)$ in Eq. (7) is substituted by $N_{p}(0) \exp \left(-k_{p} t\right)$. This simplification is valid since the larger percentage of pairs forms initially as suggested by the observed instantaneous singlet dissociation [Fig. 6(a)]. Moreover, EA quenching to nearly $80 \%$ during the first $20 \mathrm{ps}$ implies that charge pairs photogenerated in these time scales would feel the action of an electric field notably reduced, leading therefore to negligible drift. Taking into account all these considerations we can proceed to fit the experimental data and extract the values for the described parameters, which are provided in Table I.

\section{Discussion}

The effective field inside the photogenerated area and the evolution of the charge population predicted by the model are shown in Figs. 8(a) and 8(b), respectively. Field induced singlet dissociation leads to a population of photogenerated charges of which $70 \%$ of them are created within the pulse duration, while the remaining fraction is produced along the singlet lifetime, similarly as it has been reported in certain conjugated polymers. ${ }^{20-22}$ Initial dissociation is likely due to the hot state lower binding energy. As suggested by Arkhipov et al., phonons may act as a thermal bath rising the local effective temperature, thus favoring dissociation, ${ }^{23}$ a prediction which has already been experimentally confirmed in polymers such as PFO or MEH-PPV. ${ }^{24,25}$ After photogeneration, charge pairs recombine geminately and the initial charge pair population is reduced by $40 \%$ after 450 ps. How-
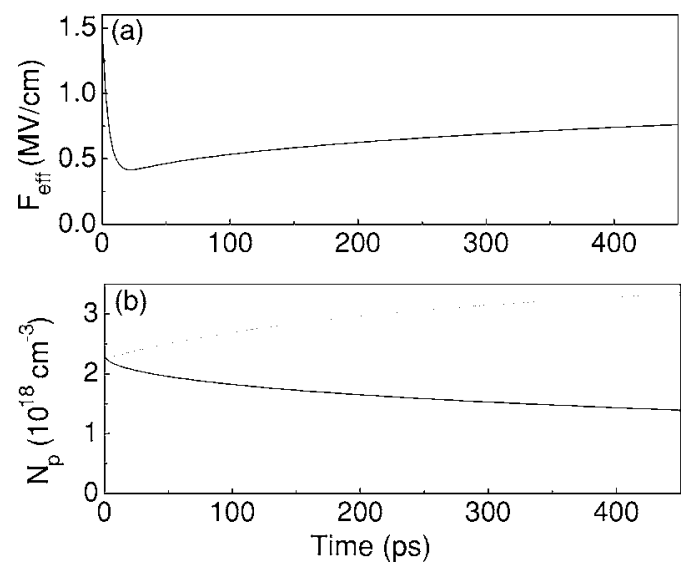

FIG. 8. Predictions from the model: (a) Evolution of the effective electric field inside the photogenerated area taking into account only the drift of the initially photogenerated $e-h$ pairs. (b) Evolution of the initial charge pair population (straight line) and total charge pair population including pairs photogenerated beyond the pulse duration (dotted line). ever, field induced singlet dissociation remains active during the entire singlet lifetime according to the $\gamma$ value employed to fit the singlet population recovery [Fig. 6(b)]. Moreover, it dominates over charge recombination leading to a progressive build up of charges up to 400 ps. Taking into account the total number of charge pairs generated after $400 \mathrm{ps}$, the fraction of pairs undergoing geminate recombination is approximately $30 \%$ of the total pair population. In addition, a $20 \%$ upper limit value for CPG quantum yield can be estimated from the values for $N_{s}(0)$ and the maximum of $N_{p}$ $\left(3.4 \times 10^{18} \mathrm{~cm}^{-3}\right.$ as displayed in Fig. 8). The kinetics of charge carriers in PCBM monitored from the nanosecond to the millisecond regime in the absence of electric field have been the object of study in a recent publication. ${ }^{26}$ In this work the population of charge carriers were generated with $2 \mathrm{~ns}$ ionizing high energy pulses and probe by monitoring changes in the microwave conductivity. It turned out that the kinetics followed stretched exponential law with a long decay component extending to the millisecond regime. This behavior was explained as due to the fact that most of the generated charge pairs produced by ionization escape from Coulombic interaction and their nongeminate recombination is controlled by a slow diffusion process. In addition to this, a subnanosecond decay component of the dielectric conductivity was also monitored and attributed to a small fraction of pairs undergoing geminate recombination. Based on the discrepancies between the $0.043 \mathrm{~cm}^{2} / \mathrm{Vs}$ mobility value estimated from $100 \%$ escaping probability with other values reported with the time-of-flight technique, it was conjectured that the geminate recombination fraction could approach $15 \%$ or more of the initial charge pair population in good agreement with our results.

Regarding charge transport, photogenerated carriers drift under the internal field with an initial velocity of 6 $\times 10^{4} \mathrm{~cm} / \mathrm{s}$ estimated from $d\langle R(t)\rangle / d t$ (see Table I), increasing therefore the average $e-h$ separation within each pair and at the same time screening progressively the internal field up to $60 \%$ of its original value. This phenomenon saturates at approximately $20 \mathrm{ps}$, when the average $e-h$ separation has reached a value of approximately $2.9 \mathrm{~nm}$ according to the expression found for $\langle R(t)\rangle$. Saturation is attributed to the reduction of the internal field which engines charge drift as well as charge trapping at interdomain boundaries in agreement with the large degree of polycrystallinity found in PCBM films. ${ }^{27}$ Beyond $20 \mathrm{ps,} \mathrm{the} \mathrm{effects} \mathrm{of} \mathrm{geminate}$ polaron-pair recombination become noticeable, being reflected in a slow recovery of the effective field which rises to $50 \%$ of its initial value after 450 ps. Our data suggest that the effects of the photoinduced polarization on the electric field could last for several nanoseconds. In spite of the numerous works reported in literature regarding nanosecond decay ki- 
netics in PCBM, ${ }^{28-30}$ we are not aware that this effect has been reported so far. We attribute it mainly to the preferential orientation of charge pairs along the field axis together with the large polaron density generated with our technique (c.a. $10^{18} \mathrm{~cm}^{-3}$ ), three orders of magnitude larger than those obtained for instance with time resolved microwave conductivity measurements.

\section{CONCLUSION}

Based in a combination of $E A$, pump probe, transient Stark spectroscopy, and field assisted pump probe we observe that about $30 \%$ of the photogenerated charge pairs recombine geminately within $0.5 \mathrm{~ns}$. Our results are complementary to previous investigations performed with microwave conductivity. Both experiments avoid measuring collected charges, then probing exclusively material properties in different time domains: ns to ms with microwave conductivity, and sub-ps to $500 \mathrm{ps}$ with our experiments. We conclude that geminate charge recombination limits significantly the charge collection efficiency in methanofullerene based solar cells.

\section{ACKNOWLEDGMENTS}

J. C.-G. acknowledges financial support from the EC under Project No. HPRN-CT-2002-00327 (EUROFET). T.D.A. is grateful to Engineering and Physical Sciences Research Council (EPSRC) and EU (EUROFET) for the financial support.
${ }^{1}$ G. Yu, J. Gao, J. C. Hummelen, F. Wudl, and A. J. Heeger, Science 270, 1789 (1995).

${ }^{2}$ N. S. Saricftci, L. Smilowitz, A. J. Heeger, and F. Wudl, Science 258, 1474 (1992).

${ }^{3}$ F. Padinger, R. S. Rittberger, and N. S. Sariciftci, Adv. Funct. Mater. 13, 85 (2003).

${ }^{4}$ X. N. Yang, J. K. J. van Duren, R. A. J. Janssen, M. A. J. Michels, and J. Loos, Macromolecules 37, 2151 (2004).

${ }^{5}$ R. Pacios, J. Nelson, D. D. C. Bradley, and C. J. Brabec, Appl. Phys. Lett. 83, 4764 (2003).

${ }^{6}$ S. A. Choulis, J. Nelson, Y. Kim, D. Poplavskkyy, T. Kreouzis, J. R. Durrant, and D. D. C. Bradley, Appl. Phys. Lett. 83, 3812 (2003).

${ }^{7}$ T. D. Anthopoulos, C. Tanase, S. Setayesh, E. J. Meijer, J. C. Hummelen, P. W. M. Blom, and D. M. De Leeuw, Adv. Mater. (Weinheim, Ger.) 16, 2174 (2004).

${ }^{8}$ T. D. Anthopoulos, D. M. De Leeuw, E. Cantatore, P. van't Hof, J. Alma, and J. C. Hummelen, J. Appl. Phys. 98, 054503 (2005).

${ }^{9}$ T. D. Anthopoulos, F. B. Kooistra, H. J. Wondergem, D. Kronholm, J. C. Hummelen, and D. M. DeLeeuw, Adv. Funct. Mater. 18, 1679 (2006).

${ }^{10}$ T. D. Anthopoulos, D. M. de Leeuw, E. Cantatore, S. Setayesh, E. J. Meijer, C. Tanase, J. C. Hummelen, and P. W. M. Blom, Appl. Phys. Lett. 85, 4205 (2004).

${ }^{11}$ J. Cabanillas-Gonzalez, T. Virgili, A. Gambetta, G. Lanzani, T. D. Anthopoulos, and D. M. De Leeuw, Phys. Rev. Lett. 96, 106601 (2006).

${ }^{12}$ T. Virgili, J. Cabanillas-Gonzalez, L. Lüer, and G. Lanzani in Photophysics of Molecular Materials, edited by G. Lanzani (Wiley-VCH, Berlin, 2005).

${ }^{13}$ D. M. Guldi, H. Hungerbühler, and K. D. Asmus, J. Phys. Chem. 99, 13487 (1995).

${ }^{14}$ D. Dick, X. Wei, S. Jeglinski, R. E. Benner, Z. V. Vardeny, D. Moses, V. I. Srdanov, and F. Wudl, Phys. Rev. Lett. 73, 2760 (1994).

${ }^{15}$ S. L. Ren, Y. Wang, A. M. Rao, E. McRae, J. M. Holden, T. Hager, K. A. Wang, W. T. Lee, H. F. Ni, J. Selegue, and P. C.
Eklund, Appl. Phys. Lett. 59, 2678 (1991).

${ }^{16}$ G. Cerullo, D. Polli, G. Lanzani, S. De Silvestri, H. Hashimoto, and R. J. Cogdell, Science 298, 2395 (2002).

${ }^{17}$ C. Gadermaier, G. Cerullo, G. Sansone, G. Leising, U. Scherf, and G. Lanzani, Phys. Rev. Lett. 89, 117402 (2002).

${ }^{18}$ C. Gadermaier, G. Cerullo, C. Manzoni, U. Scherf, E. J. W. List, and G. Lanzani, Chem. Phys. Lett. 384, 251 (2004).

${ }^{19}$ S. L. Dexheimer, W. A. Vareka, D. Mittleman, A. Zettl, and C. V. Shank, Chem. Phys. Lett. 235, 552 (1995).

${ }^{20}$ W. Graupner, G. Cerullo, G. Lanzani, M. Nisoli, E. J. W. List, G. Leising, and S. De Silvestri, Phys. Rev. Lett. 81(15), 3259 (1998).

${ }^{21}$ J. Cabanillas-Gonzalez, M. R. Antognazza, T. Virgili, G. Lanzani, C. Gadermaier, M. Sonntag, and P. Strohriegl, Phys. Rev. B 71, 155207 (2005).

${ }^{22}$ L. Lüer, H. J. Ehelhaaf, D. Oelkrug, C. Gadermaier, G. Cerullo, and G. Lanzani, Phys. Rev. B 68, 155313 (2003).

${ }^{23}$ V. I. Arkhipov, E. V. Emelianova, S. Barth, and H. Bässler, Phys. Rev. B 61, 8207 (2000).

${ }^{24}$ C. Silva, A. S. Dhoot, D. M. Russell, M. A. Stevens, A. C. Arias, J. D. MacKenzie, N. C. Greenham, R. H. Friend, S. Setayesh, and K. Mullen, Phys. Rev. B 64, 125211 (2001).

${ }^{25}$ E. Hendry, J. M. Schins, L. P. Candeias, L. D. A. Siebbeles, and M. Bonn, Phys. Rev. Lett. 92, 196601 (2004).

${ }^{26}$ M. P. de Haas, J. M. Warman, T. D. Anthopoulos, and D. M. De Leeuw, Adv. Funct. Mater. 16, 2274 (2006).

${ }^{27}$ C. J. Brabec, N. Sariciftci, and J. C. Hummelen, Adv. Funct. Mater. 11, 15 (2001).

${ }^{28}$ A. F. Nogueira, I. Montanari, J. Nelson, J. R. Durrant, C. Winder, N. S. Sariciftci, and C. Brabec, J. Phys. Chem. B 107, 1567 (2003).

${ }^{29}$ T. J. Savenije, J. E. Kroeze, M. M. Wienk, J. M. Kroon, and J. M. Warman, Phys. Rev. B 69, 155202 (2004).

${ }^{30}$ S. C. J. Meskers, P. A. van Hal, A. J. H. Spiering, J. C. Hummelen, A. F. G. van der Meer, and R. A. J. Janssen, Phys. Rev. B 61, 9917 (2000). 